\title{
DIVERGENCIAS SIGNIFICATIVAS ENTRE LA FORMACIÓN ACADÉMICA Y EL PERFIL PROFESIONAL EN LA ESPECIALIDAD DE MAESTRO EN LENGUA EXTRANJERA
}

\author{
Ma José Bonachía Caballero \\ Julieta Ojeda alba \\ $\mathbf{M}^{\mathrm{a}}$ Luz Oyón Bañales \\ Universidad de La Rioja
}

\begin{abstract}
RESUMEN: Este breve estudio Ilama la atención sobre las desconexiones que existen entre los programas de Maestro en L.E. y las necesidades profesionales de los futuros maestros. Tanto las actuales tendencias en la enseñanza de idiomas como las recomendaciones del M.E.C. aconsejan una exposición temprana de los aprendices a la lengua meta. Para poder llevar a cabo la docencia en estos niveles de acuerdo con dichas orientaciones, los profesores deben tener una competencia de comunicación específica.

En nuestra opinión los profesores de Infantil y Primaria no están formados para desenvolverse adecuadamente en el aula de idioma extranjero. En este artículo, sugerimos una propuesta de modificación de los programas del actual plan de estudios, ilustrándola con la puesta en práctica de dos sencillos juegos infantiles, uno en francés y otro en inglés.
\end{abstract}

ABSTRACT: This brief paper calls attention to the current disconnection that exists between the recommendations of the Spanish Department of Education and the programs currently in use in the Spanish Escuelas de Magisterio. The present tendencies of second language teaching in Europe recommend an early exposure of students to the target language (kindergarden and first years of primary school), but in order to teach children at this level teachers must master a type of classroom speech which is often ignored in foreign language instruction at university level. In our opinion primary school teachers are not well trained to effectively communicate with very young students. So the only alternative for Spanish foreign language teachers is to carry out a traditional formal teaching of the language which is not the best way to motivate young students. The ultimate responsibility in this situation lies with professors in the Escuelas de Magisterio.

In order to demonstrate these deficiencies we here use as an example the implementation of two simple child games. 
PALABRAS CLAVE: Perfil profesional, programa de estudios, formación de profesores, enseñanza de lenguas, segunda lengua extranjera

KEYWORDS: Professional profile, studies program, teacher training, language teaching, second language teaching

Las directrices europeas en materia de enseñanza de L.L.E.E. aconsejan una exposición a la lengua meta cada vez más temprana y, obedeciendo a este mismo principio, las normativas ministeriales establecen la enseñanza de L.L.E.E. desde el primer ciclo de Primaria. Sin embargo, un somero análisis de nuestro actual Plan de Estudios de Maestro en L.L.E.E. evidencia divergencias significativas entre la formación actual del alumno de Magisterio y el perfil profesional adaptado a estas demandas. Partiendo esencialmente de nuestra experiencia docente, y motivadas por la esperanza de colaborar con la solución a estos problemas, hemos reflexionado sobre los contenidos de estos planes de estudio y hemos llegado a la conclusión de que, al margen de otros posibles obstáculos, los alumnos de Magisterio en lengua extranjera (Francés/Inglés), no abandonan las aulas con la formación adecuada para cumplir su función: enseñar una segunda lengua a niños de entre seis y doce años.

Nuestros alumnos tienen a lo largo de los tres cursos de su diplomatura únicamente tres cuatrimestres de lengua: con estos dieciocho créditos (180 horas de clase presencial) se pretende dotar a los futuros maestros de un conocimiento de la lengua suficiente para hacer frente con seguridad y dominio al contexto de la clase de Inglés/Francés en el aula de Primaria y de Enseñanza Precoz. Nuestra opinión personal es que esta carga lectiva es a todas luces insuficiente para preparar al profesor de infantil y primaria de L.L.E.E. No obstante, no es este el foro donde se puede discutir y solucionar la necesidad de ampliar el creditaje y, por tanto, nos limitaremos a discutir la necesidad y posibilidad de optimizar los resultados contando con el número de créditos actuales. Si analizamos los programas de Idioma, encontramos diferentes temas en los que se proponen nociones y funciones muy amplias en torno a la descripción, narración, argumentación del discurso etc. elaborados a partir de bloques temáticos referidos a cuestiones de actualidad o de la vida cotidiana que proporcionan al futuro maestro de L.L.E.E. contenidos comunicativos y socio-culturales muy generales.

Sin embargo, como vamos a tratar de mostrar, los programas en vigor no tienen una relación directa con el contexto escolar del ciclo de Enseñanza Precoz y Primaria como sería necesario para la formación profesional de estos docentes. Esta falta de conexión no es nueva, hace ya más de veinte años que Glyn S. Hughes observó: "A pesar de las demandas lingüísticas de una situación de enseñanza de L2, los diplomados o licenciados en L.L.E.E. raramente están adecuadamente formados para la tarea, aparentemente simple, de enseñar L.L.E.E."1 Para este autor, como para

1. Todas las traducciones incluídas en este trabajo han sido realizadas por las propias autoras.

"Despite the linguistic demands of the L2 teaching situation, foreign language graduates are seldom adequately prepared for the seemingly simple task of running a class in the L2", A Handbook of Classroom English, pág. 82. 
muchos otros que han escrito sobre este tema en años posteriores, la misma naturaleza de los temarios es a veces la que limita el tiempo que se debería dedicar a practicar las funciones clave para el correcto desarrollo de una clase de L.E. en estos niveles, utilizando la lengua extranjera como medio de comunicación. Uno de los resultados de esta desconexión entre la formación académica y las exigencias profesionales es, como de nuevo observa Hughes, que al finalizar sus estudios "el alumno de Magisterio domina un repertorio muy limitado del discurso en el aula y hace un uso mínimo de la L2". ${ }^{2}$ Esta falta de competencia comunicativa en el discurso del aula empobrece y limita en gran medida el input que reciben los aprendices y, en consecuencia, su adecuado avance.

Esta disociación resulta aún más sorprendente si tenemos en cuenta que las orientaciones del M.E.C. ya desde 1992 instan a que "los contenidos de Lengua Extranjera sean contextualizados en la experiencia del niño". ${ }^{3}$ El M.E.C., con un enfoque claramente constructivista, insiste en que el maestro debe partir necesariamente del conocimiento previo del niño para que éste entre en contacto con un input lingüístico que no le es familiar pero sí comprensible en la medida en que se aproxima a sus propias vivencias. En definitiva, se pretende poner en práctica la idea que S. D. Krashen y otros teóricos han expuesto sobre la necesidad de facilitar el aprendizaje a partir de un input comprensible. ${ }^{4}$

Por otro lado, una de las propuestas del Consejo de Educación europeo propone instrumentalizar la L.E. utilizándola como medio de comunicación para enseñar otras materias, planteamiento apoyado por numerosos autores desde distintos ámbitos, subrayando así los buenos resultados a partir de la instrumentalización del idioma. ${ }^{5}$ En efecto, en la medida en que se presenta la L.E. no como un fin en sí misma, sino como un medio para realizar otras actividades escolares (plástica o expresión corporal, por ejemplo) que el niño percibe como meta, se propicia una implicación afectiva y cognitiva mayor. De esta forma se desvía la atención del objetivo lingüístico para dirigirla hacia el contenido semántico de la actividad.

Instrumentalizar el idioma implica utilizarlo no como un fin en sí mismo, sino como una herramienta con la que se hacen cosas además de aprender el idioma de manera más o menos inconsciente: el objetivo es lograr que los niños participen activamente en el uso del lenguaje. Este enfoque significa que el maestro de Enseñanza Precoz y Primaria debe diseñar actividades que permitan "usar" el idioma con sinceridad en el aula y crear un contexto compartido, reconocido por todos, en palabras de Zanón "crear un motor afectivo para aprender lo que no saben". ${ }^{6}$ Estos prin-

2. "the trainee teacher acquires a very limited repertoire of classroom phrases, or makes as little use of the L2 as possible", A Handbook of Classroom English, pág. 5.

3. Libro Rojo del M.E. C.

4. Véase Second Language Acquisition and Second Language Learning. Es importante hacer notar que estas ideas constructivistas no son nuevas sino que han sido repetidas a lo largo de la historia. Sin remontarson más atras ya el filósofo y escritor Henry David Thoreau observó en su ensayo Walden: "We only learn what we half know" (sólo aprendemos lo que ya conocemos a medias).

5. Véase Ch. O’Neil, 1983; H.G.Widdowson, 1981 y J.M. Artigal, 1990

6. Veáse Zanón, "Cómo no impedir que los niños aprendan inglés", Comunicación lenguaje y educación, pág. 98. 
cipios básicos han servido para que la enseñanza/aprendizaje de idiomas ofrezca una amplia gama de actividades adaptadas a la experiencia del niño y a la posibilidad de utilizar la L.E como un medio.

Coherentes con estos planteamientos, la gran mayoría de los manuales de inglés/francés para niños que hemos analizado, ${ }^{7}$ ofrecen una serie de propuestas tales como juegos, trabajos de plástica, cuentos, canciones, tiras cómicas y resolución de problemas (enigmas, adivinanzas, etc.), actividades muy relacionadas con el mundo de los niños y que responden al enfoque constructivista que, como hemos observado anteriormente subyace en los principios de la enseñanza de lenguas extranjeras. Ahora bien, el problema se manifiesta cuando el maestro, que no ha sido convenientemente formado, intenta poner en práctica esta serie de actividades. Este hecho, como ya hemos apuntado, es el resultado de la disociación existente entre los contenidos de las asignaturas de idioma del Plan de estudios de Maestro en L.E. y el contexto escolar.

Esta disociación ha sido también identificada por los alumnos de prácticas de tercer curso de Magisterio y reflejada a través de una encuesta, adjunta en anexo, realizada con el propósito de sondear su opinión respecto a la pertinencia de los programas de idioma en la diplomatura que están finalizando. La encuesta arroja resultados reveladores:

El $100 \%$ de los alumnos encuestados considera que es necesario conocer la lengua específica para llevar a cabo juegos y actividades de plástica en la escuela.

Un 70\% aproximadamente admite que no conoce ningún tipo de juego apropiado para el ciclo de Primaria, ni tampoco se considera capacitado para ponerlo en práctica en el aula utilizando la lengua extranjera como instrumento de comunicación. El recuento final de las respuestas a las cuatro secciones de esta pregunta presenta un panorama preocupante: este mismo porcentaje desconoce el léxico necesario para referirse a los materiales y carece de recursos lingüísticos para explicar las reglas y organizar la interacción de los alumnos. Estos conocimientos son indispensables para la realización de este tipo de actividades.

Alrededor de un $80 \%$ considera que no existe una adecuación entre los contenidos de los programas mencionados y las actividades que se desarrollan en el aula de Primaria y enseñanza Precoz.

Casi un $100 \%$ de los alumnos han seleccionado las dos opciones propuestas en la encuesta: mayor contacto con los materiales de Primaria y la práctica en clase de L.E. de situaciones de comunicación utilizadas en el contexto escolar.

Como profesores de L.E. de Magisterio nos concierne, más que a ningún otro profesional de la educación, evitar estas divergencias y ser coherentes con las orientaciones actuales, adaptando los contenidos y la metodología de los programas vigentes al perfil profesional que se exige de nuestros estudiantes. Por tanto, nos parece imprescindible incluir en nuestros programas contenidos coincidentes con la enseñanza/aprendizaje de la L.E. en la Escuela. Es necesario que entre estos contenidos figuren aquellos que puedan proporcionar a nuestros alumnos una competencia

7. Manuales de inglés: Fanfare, English Club, Boomerang, Big Red Bus y Road to English etc.

Manuales de Francés: Abracadabra, Farandole, Kangourou Trampoline, La Baguette Magique etc. 
comunicativa específica para desenvolverse con éxito en el aula de L.L.E.E. tal como se entiende hoy.

Partimos de la base de que el maestro debe poseer una competencia lingüística específica para estos niveles de enseñanza muy por encima de las posibles demandas de sus alumnos. No se trata, evidentemente, de que sepa comentar una obra literaria de Shakespeare, o un tema científico como el de los residuos nucleares, sino de que tenga los conocimientos requeridos para saber explicar, entre otras cosas: cómo se juega en inglés a "hide-and-seek" (el escondite), o en francés a "les chaises musicales" (sillas musicales); cómo contar un sencillo cuento infantil; cómo dar instrucciones para realizar un trabajo manual o un juego. En resumen, el maestro de L. E. debe dominar especialmente el léxico y los actos de habla relativos al mundo de la infancia.

El dominio de este léxico específico es especialmente relevante en la formación de los maestros de L.E. por la facilidad con la que los niños aprenden vocabulario, idea corroborada desde diferentes puntos de vista por varios autores. Así, Ch. O'Neil opina que los niños son particularmente sensibles a la adquisición léxica. Numerosos estudios realizados sobre adquisición de lenguaje inciden en el hecho de que son precisamente las palabras léxicas las que con mayor rapidez se incorporan al vocabulario activo del aprendiz. Para Krashen, más vocabulario significa más comprensión del input que, a su vez, conlleva más adquisición de la sintaxis de una forma casi inconsciente. En esta misma línea, W. Klein opina que aunque las palabras léxicas aparezcan con mucha menos frecuencia dentro del discurso, éstas se adquieren mucho antes que las gramaticales, debido a la entonación y a la información paralela que las acompaña. ${ }^{8} \mathrm{~J}$. Courtillon considera igualmente que las palabras léxicas son las que aportan una mayor información y contribuyen así en gran manera a facilitar la comunicación entre los alumnos en los primeros estadios de aprendizaje.

Así pues, con el fin de propiciar este conocimiento léxico, el futuro maestro debe manejar una amplia variedad de documentos auténticos orales y escritos cercanos a la experiencia del niño: revistas infantiles como Astrapi, Tobogan, Pomme d'Api, Charade, Stone Soup, Barney, Boy's Life, etc.; juegos tradicionales como los franceses "carte de visite", "Jacques a dit", "la marelle", "I'aveuglette", "jouer à chat", o los ingleses "Simon says", "Guess who?", "Twenty Questions", "Hangman" etc.; cuentos populares como los franceses Cendrillon, Le chat botté, Blancheneige, Les fées o los ingleses Goldie Locks, Jack and the Bean Stalk etc.; películas infantiles, canciones y un largo etcétera.

Además de esta competencia lingüística evidentemente el maestro debe dominar el resto de las competencias necesarias: competencia sociolongüística, discursiva, estratégica etc., para facilitar la comunicación en el aula. El dominio de todas estas competencias facilitaría la puesta en práctica de actividades tales como pasar lista, dar instrucciones diversas, asignar tareas, informar sobre normas de funcionamiento, etc. en las que la L.E. sería el instrumento de comunicación propiciando un significativo aumento del input. Numerosos autores coinciden en señalar que el discurso que se deriva de este tipo de prácticas en el aula es el que facilita un marco natural para la adquisición del idioma extranjero. Así, Claire Kramsch señala que la

8. Véase W. Klein, L'acquisition de langue étrangère, pág. 94-98. 
clase de L.E. es el lugar de encuentro de dos tipos de discurso: el discurso en L.E. que constituye el contenido del aprendizaje y que los alumnos tratan de adquirir y, por otro lado, el discurso que sirve para organizar este aprendizaje, es decir, el diálogo entre maestro y alumnos, didáctico en ciertos momentos, 'natural' en otros. ${ }^{9}$

En nuestra opinión, todas estas carencias comunicativas a las que aludimos, quedan expuestas al poner en práctica dos sencillos juegos muy conocidos entre los niños: uno en francés "colin-maillard" y otro en inglés "donkey". La correcta y efectiva realización de estos juegos en el aula implica el dominio de las competencias a las que nos hemos referido.

Comenzamos por el juego de "colin-maillard". Para realizarlo, y siguiendo las pautas anteriores, el maestro debe conocer un léxico específico: Vendarse los ojos, dado, cubilete, dar vueltas, hacer un corro, echar a suertes, etc. así como saber transmitir las consignas necesarias para la puesta en práctica del juego, enunciando una serie de actos de habla tales como:

1. Hoy vamos a jugar a la gallinita ciega.

2. Separad los pupitres, y todas vuestras cosas.

3. Haced un corro y dejad libre el centro de la clase.

4. Os voy a explicar cómo se juega a la gallinita ciega.

3. Vamos a echar a suertes a ver a quién se la queda.

4. " $X$ ", dame el dado y el cubilete.

5. Ahora, vamos a tapar los ojos a "Y".

6. El jugador " $Y$ " va a intentar pillaros mientras os movéis por la clase.

7. Cuando coja a alguien, tiene que identificarle diciendo en francés como se Ilama.

8. Si acierta, se libera y el desenmascarado ocupa su lugar.

Del mismo modo, para poner en práctica el popular juego de cartas "donkey" (burro) el maestro tiene que conocer el léxico específico de los juegos de cartas: jugar a las cartas, barajar, jugador, formar parejas robar una carta, tirar una carta, descartarse, poner la carta boca arriba, boca abajo, esperar el turno, comodín etc. y los actos de habla necesarios tanto para organizar previamente la clase como para poner en práctica el juego:

1. El objetivo del juego es reunir cuatro cartas iguales lo antes posible.

2. El niño que tiene la mano baraja las cartas.

3. Reparte cuatro cartas a cada jugador poniéndolas boca abajo sobre la mesa.

4. Cuando todos los jugadores tienen sus cartas el que está de mano dice "ya".

5. Cada jugador elige una carta que no quiere y la pasa al jugador que está a su izquierda

6. Cada jugador mira con rapidez la carta que ha recibido y decide si la pasa o la guarda. 
7. Si un jugador se guarda la carta que ha recibido debe pasar otra.

8. Los jugadores deben pasar las cartas con rapidez.

9. Tan pronto como un jugador tiene cuatro cartas iguales se coloca un dedo a un lado de la nariz procurando no ser visto por los demás.

10. Los jugadores que lo ven lo imitan.

11. El último en hacerlo es "burro" y se pone la b de burro en una lista donde se han escrito los nombres de todos los jugadores.

12. Ahora el jugador que está a la izquierda del de mano, baraja y da las cartas.

13. Tan pronto como dice "ya" el juego empieza de nuevo.

14. El jugador que pierda primero cinco veces (el número de letras de burro) es nombrado "el peor jugador" y el que tiene menos letras es nombrado "mejor jugador" y gana el juego.

Estos dos juegos son extremadamente simples, teniendo en cuenta que los niños los practican a menudo en su lengua materna; sin embargo, una lectura cuidadosa revelará las dificultades que sin duda tendría el mejor de nuestros diplomados para ponerlo en práctica y dar a los niños las explicaciones pertinentes con expresiones correctas. Nuestros estudiantes simplemente no dominan el léxico necesario para producir estos actos de habla y, por lo tanto, ninguna de las siguientes expresiones serían producidas con naturalidad ni en francés ni en inglés: "Mettez -vous en cercle", "on va tirer au sort qui va être le premier joueur", "on va bander les yeux de untel", "on va se mettre autour de lui", "on le frôle avec la main", etc. (francés); o "shuffle a deck of cards", "the dealer starts", "deal out"; "place the deck facedown", "it's his/her turn", "a set of four matching cards", etc. (inglés).

Aceptamos como probable que, si el maestro es imaginativo, pueda emplear estrategias de comunicación para paliar el desconocimiento de algunas de estas expresiones y desenvolverse airosamente ante los niños aunque desconozca el léxico específico. Sin embargo, se trata de que el input aportado por el profesor sea modélico tanto por su corrección como por su riqueza léxica ya que estas carencias tienen consecuencias extraordinariamente negativas para los pequeños aprendices. Por ejemplo, un profesor que desconoce la expresión "shuffle the deck" (baraja las cartas), podría enseñar a decir "move the cards", "stir the cards" o una forma similar, pero este procedimiento que demostraría que un aprendiz tiene estrategias comunicativas, no es disculpable en un enseñante. La otra opción que se le presenta al profesor sin una adecuada formación, es simplemente no poner en práctica en la clase ningún juego o actividad para la que no está preparado, limitando necesariamente su metodología a la enseñanza formal de la lengua. Los profesores de lenguas extranjeras que tutorizamos el prácticum sabemos que esta situación es más frecuente en la enseñanza de idiomas de lo que sería conveniente, precisamente porque algunos profesores de Primaria tienen conciencia de sus propias limitaciones.

Por consiguiente, la necesidad de modificar esta situación es perentoria si queremos adaptarnos al perfil profesional de nuestros alumnos. Nuestra propuesta de programación de contenidos específicos se articularía en torno a "tareas" para cuya realización consideramos la siguiente secuencia: 
1) Oferta de una amplia lista de los juegos y actividades (plástica, expresión corporal, etc.) más conocidos entre los niños de Infantil y Primaria.

2) Negociación, selección y secuenciación de juegos y actividades a realizar según su nivel de complejidad, comenzando por aquellos dirigidos a Enseñanza Precoz para continuar con los más adecuados para los alumnos de Primaria.

3) Recogida y toma de contacto con los materiales necesarios para su realización.

4) Puesta en común del léxico y de los actos de habla para llevar a cabo los juegos o actividades elegidas.

5) Producción por los alumnos, en grupos pequeños, de textos en los que se recojan las instrucciones y el discurso del profesor en el aula para realizar dichos juegos y actividades.

6) Realización en clase de la actividad o juego en el que previamente ha trabajado cada grupo.

7) Prácticas de los alumnos de magisterio con alumnos de Enseñanza Precoz y Primaria. Grabación y posterior sesión de observación y discusión de la misma.

Esta puesta en práctica ofrecería algunas ventajas; en primer lugar, la propuesta de un programa en el que contenidos y procesos formasen un continuum; $;^{10}$ en segundo lugar, la coherencia del planteamiento con las últimas propuestas europeas sobre enseñanza de L.L. E.E. en la medida que posibilitaría un aprendizaje del inglés/francés realmente coherente con el perfil profesional de nuestros alumnos y, finalmente, una integración real de los contenidos de lengua y de su didáctica.

Se podría objetar con razón que, para que un maestro pueda poner en práctica la enseñanza que propugnamos necesitaría un dominio lingüístico equivalente como mínimo al de los licenciados. Tradicionalmente se ha asumido que el profesor de lengua extranjera de Secundaria debe poseer más competencia comunicativa y mayores destrezas que el de Primaria y Enseñanza Precoz. Estamos totalmente en desacuerdo con esta presunción y nos atrevemos a sostener justamente lo contrario. Es obvio que el léxico, las construcciones y fonemas que el maestro de Primaria emplee en la clase van a marcar más profundamente a sus alumnos que las que utiliza el profesor en Secundaria. Entre los psicolingüistas está extendida y fundamentada la idea de que precisamente entre los ocho y los doce años disminuye la plasticidad del cerebro y la capacidad de adquirir una segunda lengua, como observan Heidi Dulay y otros." Aceptada esta premisa, cualquier explicación adicional de las razones por las que el profesor de Primaria debe poseer una competencia igual o superior que el de

10. Utilizamos continuum en el sentido de "dominio de los contenidos necesarios para la comunicación/desarrollo de los procesos subyacentes a las actividades de comunicación" según J. Zanón y otros.

11. "Children under ten who experience enough natural communication in the target language nearly always succeed in attaining native-like proficiency, while those over fifteen rarely do". (H. Dulay y otros, 1982:78). 
Secundaria es superflua. ${ }^{12}$ En conclusión, nos corresponde a los profesores de Magisterio tomar conciencia clara de la divergencia existente entre nuestros programas y la escuela y por tanto adaptar estos programas ofreciendo a nuestros alumnos los instrumentos adecuados para realizar su trabajo debidamente. De esta manera, nos ajustaríamos tanto a las demandas sociales como a las propuestas europeas en materia de enseñanza/aprendizaje de lenguas.

\section{BIBLIOGRAFÍA}

ARTIGAL J.M. (1990) Uso/Adquisición del lenguaje como construcción de territorio compartido. En Ponencias y Comunicaciones del XVI Congreso de AELFA. Madrid: Inserso, Ministerio de Asuntos Sociales.

CASTELLOTI, V. y DE CARLO, M. (1995) La formation des enseignants de langue. París: Clé International.

COURTILLON, J. (1989) Lexique et apprentissage de la langue. En Lexiques. Paris: Hachette.

DULAY, H. y otros (1982) Language Two. Oxford: Oxford University Press.

HEATON, J.B. (1981) Using English in the Classroom: A Functional Approach for Teachers. London: Longman.

HUGHES, S.G. (1981) A Handbook of Classroom English. Oxford: Oxford University Press.

KLEIN, W. (1989) L'acquisition de langue étrangère. Paris: A. Colin.

KRAMSCH, C. (1984) Interaction et discours dans la classe de langue. Paris: Hatier.

KRASHEN, S.D. (1981) Second Language Acquisition and Second Language Learning. Oxford: Pergamon.

O'NEIL, CH. (1993) Les enfants et l'enseignement des langues étrangères. Paris: LAL, Hatier.

PENFIELD, V. y ROBERTS, L. (1959) Speech and Brain Mechanisms. Princeton, NJ: Princeton University Press.

Primaria (Caja Roja) (1992) Madrid: M.E.C.

WIDDOWSON, H.G. (1978) Teaching language as communication. Oxford: Oxford University Press.

WILLIS, J. (1981) Teaching English Through English. London: Longman.

ZANÓN, J. (1992) Cómo no impedir que los niños aprendan inglés. En Comunicación, lenguaje y educación, 16, 93-109.

ZANÓN, J. (1999) La enseñanza del español mediante tareas, Madrid: Edinumen.

12. Véase V. Penfield y L. Roberts, Speech and Brain Mechanisms. 


\section{ANEXO}

\section{ENCUESTA PARA LOS ALUMNOS DE MAGISTERIO (LENGUA EXTRANJERA)}

1. ¿Consideras necesarios los juegos y actividades de plástica para el aprendizaje de idioma en el ciclo de Primaria? si--no

2. ¿Conoces juegos infantiles y/o actividades manuales realizables en el primer ciclo de Primaria? (8-10 años). si--no. Enuméralas:

3. Te consideras capacitado para realizar alguna de estas actividades utilizando el inglés/francés como instrumento de comunicación? si--no

4. Si tuvieras que realizar alguna de estas actividades ¿qué carencias lingüísticas destacarías?
a) Desconocimiento de los juegos y actividades
b) Desconocimiento del léxico referido a materiales y útiles para jugar.
c) Falta de recursos lingüísticos para comunicar reglas y consignas.
d) Falta de recursos lingüísticos para organizar la interacción verbal del grupo.

5. ¿Qué soluciones aportarías para subsanar las posibles deficiencias lingüísticas?
a) Mayor contacto en clase de lengua con materiales de primaria, tales como, canciones, juegos, videos etc.?
b) Práctica en clase de lengua de situaciones de comunicación para realizar este tipo de actividades

6. ¿Consideras que hay relación entre los contenidos de los programas?

de Idioma I, II, III de Magisterio y la práctica de las actividades arriba citadas? 